\title{
Unexpected diversity of CRISPR unveils some evolutionary patterns of repeated sequences in Mycobacterium tuberculosis
}

(1)

\author{
Guislaine Refrégier $^{1 *}$, Christophe Sola ${ }^{1 *}$, Christophe Guyeux ${ }^{2}$.
}

1: Institute for Integrative Biology of the Cell (I2BC), CEA, CNRS, Univ. Paris-Sud, Université Paris-Saclay, 91198, Gif-sur-Yvette cedex, France

2: FEMTO-ST Institute, UMR 6174 CNRS, DISC Computer Science Department, Univ. Bourgogne Franche-Comté (UBFC), 16 Route de Gray, 25000 Besançon

(1)

12

3

14

15

6

17

8

9

*co-corresponding authors : guislaine.refregier@u-psud.fr, Christophe.sola@i2bc.paris$\underline{\text { saclay.fr }}$ 


\section{Abstract}

31 Diversity of the CRISPR locus of Mycobacterium tuberculosis complex has been studied since 1997 for molecular epidemiology purposes. By targeting solely the 43 spacers present in the two first sequenced genomes ( $\mathrm{H} 37 \mathrm{Rv}$ and $\mathrm{BCG})$, it gave a biased idea of CRISPR diversity and ignored diversity in the neighbouring cas-genes.

We set up tailored pipelines to explore the diversity of CRISPR-cas locus in Short Reads. We analyzed data from a representative set of 198 clinical isolates as evidenced by wellcharacterized SNPs.

We found a relatively low diversity in terms of spacers: we recovered only the 68 spacers that had been described in 2000. We found no partial or global inversions in the sequences, letting always the Direct Variant Repeats (DVR) in the same order. In contrast, we found an unexpected diversity in the form of: SNPs in spacers and in Direct Repeats, duplications of various length, and insertions at various locations of the IS6110 insertion sequence, as well as blocks of DVR deletions. The diversity was in part specific to lineages. When reconstructing evolutionary steps of the locus, we found no evidence for SNP reversal. DVR deletions were linked to recombination between IS6110 insertions or between Direct Repeats.

This work definitively shows that CRISPR locus of $M$. tuberculosis did not evolve by classical CRISPR adaptation (incorporation of new spacers) since the last most recent common ancestor of virulent lineages. The evolutionary mechanisms that we discovered could be involved in bacterial adaptation but in a way that remains to be identified.

\section{Introduction}

Since the rise of molecular biology, repeated sequences (CRISPR, IS, VNTRs) have been used to track relatedness between individuals (Garcia De Viedma and Perez-Lago, 2018). Indeed, they share two major features essential for diversity studies: ease of study, and rapid mutation rate (van Belkum, et al., 1998). In pathogens like Mycobacterium tuberculosis complex (MTC) they have been used for molecular epidemiology, complementing contact tracing, and/or identifying unsuspected links (Garcia De Viedma and Perez-Lago, 2018). In the last 5 years however, popularity of most repeated sequences has decreased first because they are larger than reads provided by Short Reads Sequencing, and second because of the generalization of Whole-Genome-Sequence availability and use of softwares analyzing Single 
Nucleotide Polymorphisms (SNPs) (Jajou, et al., 2018; Schurch, et al., 2010). In fact, some of these repeated sequences have sufficient variation to characterize them based on reads. The boom of Whole Genome Sequencing provides plenty of data to dig into for evolutionary studies and changes the way drug-susceptibility testing will be done in the future (Consortium, et al., 2018; Mulholland, et al., 2019). We will show in the case of CRISPR sequences how this diversity can reveal unexpected evolutionary patterns. We will show in addition that in the species of focus, namely MTC, there has been no new spacers acquisition for at least 5,000 years, i.e. no adaptative evolution in the common CRISPR terminology despite the presence of Cas genes.

CRISPR acronym stands for Clustered Regularly Interspaced Short Palindromic Repeats (Jansen, et al., 2002). They are characterized by repeats of 21 to $37 \mathrm{nt}$ called Direct Repeats (DR) and the presence of unique sequences, called spacers, between each DR copy. Blocks of one DR and the following spacer has been termed Direct Variable Repeat (DVR) (Groenen, et al., 1993). CRISPR loci were first identified in Escherichia coli (Ishino, et al., 1987), their role in bacterial immunity was suspected in Yersinia pestis (Pourcel, et al., 2005), and later demonstrated in Streptococcus thermophilus (Barrangou, et al., 2007). Their presence has been detected in around 50\% percent of eubacteria and 90\% of archaebacteria (Couvin, et al., 2018; Grissa, et al., 2008; Grissa, et al., 2007; Grissa, et al., 2007). Various classes of CRISPR systems have been described (Makarova, et al., 2015). They all share the same mechanism of spacer acquisition, inserting part of a foreign sequence designated as protospacer, with a length similar to that of the repeats, next to the 5' end of the locus. In Salmonella enterica for instance, the exploration of CRISPR diversity has shown that sequences including several DVR could be deleted, and that mutations could occur in spacers (Fabre, et al., 2012), however, the increased CRISPR dictionary as well as the restricted number of genomes sequenced reduced the possibility to have an extensive understanding of their evolutionary mechanisms.

Mycobacterium tuberculosis complex (MTC) is the agent of mammal tuberculosis, with human-adapted lineages being the most diverse and well spread. Its emergence and diversification dates back to at least 5,000 years old. There are six main and widely spread human-adapted sublineages referred to as L1 to L6 and an animal-adapted lineage (Coll, et al., 2014; Gagneux, 2012; Hershberg, et al., 2008), as well as a few rare and endemic human lineages (L7, L8, L0) (Blouin, et al., 2012; Ngabonziza, et al., 2019). Their diversity is being 
93

progressively unveiled through extensive WGS (Coll, et al., 2014; Palittapongarnpim, et al., 2018; Shitikov, et al., 2017).

M. tuberculosis reference clinical isolate $\mathrm{H} 37 \mathrm{Rv}$ as well as most $M$. tuberculosis isolates carry a CRISPR locus together with a complete cas genes set of type III-A (Makarova, et al., 2015). Rare isolates lack part of CRISPR and or cas genes (Freidlin, et al., 2017). Partial analysis of the CRISPR diversity has been used since 1997 to explore the clinical isolates relatedness through a technique coined as «spoligotyping ( Kamerbeek, et al., 1997). In this technique, the presence of 43 spacers identified in $\mathrm{H} 37 \mathrm{Rv}(\mathrm{n}=35)$ or in $M$. bovis $\mathrm{BCG}(\mathrm{n}=8)$ are looked for. This results in a barcode that can be easily shared and stored. Spoligotyping has led to the set-up of the first worldwide database for this pathogen counting today more than 111,000 patterns originating from 169 countries (Couvin, et al., 2018). The absence in some isolates of individual or consecutive spacers has revealed the possibility for small and large deletions of adjacent DVR (Brudey, et al., 2006; Filliol, et al., 2003). Large deletions proved good markers of tuberculosis diversification (Comas, et al., 2009; Kato-Maeda, et al., 2011).

Extensive MTC CRISPR structure has been previously explored in 19 M. tuberculosis clinical isolates belonging to EAI (L1), Beijing (L2), Euro-American (L4) lineages, 5 from animal species M. bovis and M. microti, and one M. canettii (van Embden, et al., 2000). This work showed that additional diversity exists in the form of DR variants, and duplication of DVR. It also documented the presence of insertion sequence IS6110 in two different positions and orientations in L2 and L4 lineages. CRISPR diversity however remains unexplored in many sublineages as well as in major lineages such as L3, L5 and L6.

We recently set up a pipeline to reconstruct reliably CRISPR locus of $M$. tuberculosis (Guyeux, et al., 2019a). We selected Short Reads Archives (SRA) from the more than 60,000 available today to represent clinical isolates diversity and derived their CRISPR locus structure. The specific questions we tackled are: does MTC CRISPR locus contain additional spacers in addition to the 68 spacers ones described? What are the other patterns of diversity in CRISPR-Cas locus? What kind of underlying mechanisms of evolution can account for the observed diversity? Did the main lineages evolve similarly or are they CRISPR features specific of some lineages and/or sublineages? What is the most likely CRISPR sequence of tuberculosis most recent common ancestor (MRCA)? 


\section{Methods}

\section{Data collection}

128 One hundred ninety-eight $(\mathrm{n}=198)$ Sequence Reads Archives obtained by paired-end 129 sequencing with Illumina technology were selected from a local database of more than 3,500 130 genome sequences based on their representativeness of $M$. tuberculosis lineages (Guyeux, et 131 al., 2019a). Namely, the following numbers of data were included for each lineage: 55 for 132 Lineage 1, 20 for Lineage 2, 17 from Lineage 3, 60 from Lineage 4, 25 from Lineage 5, 7 133 from Lineage 6, 10 from Lineage 7, 1 from M. bovis, 1 from M. caprae, 1 from M. microti, 1 from $M$. pinnipedii. Data were downloaded as fasta files to decrease storage space as erroneous sequence will be ignored in the analytic steps.

\section{Identification and cataloging of CRISPR subsequences of interest}

We first looked for spacer variants by searching for patterns made up of the last 12 nucleotides of most common DR sequence and later referred to as DR0 (Kamerbeek, et al., 1997), followed by 10 to 70 nucleotides, followed by the first 12 nucleotides of the DR0. The resulting subsequences were compared to the reference spacers to be declared either as a new spacer or a variant of a known spacer (for more details; see (Guyeux, et al., 2019a)). We then used this enhanced catalogue of spacers to find DR variants, in the same way as above. The new DRs thus obtained were used for a second phase of discovery of spacers, as described above.

To the collection of different spacers and DR, we added the following subsequences of interest to be discovered in the CRISPR loci: 1) the beginning and end sequences of IS6110 and its reverse complement (40 bp each time); 2) those corresponding to $R v 2816 c$ (Cas2 gene of the Cas locus) and $R v 2813 c$, reputed to 150 border the CRISPR locus;

1513 3) the sequences found between these bordering genes and first or last DR;

152 4) the beginning and the end of each Cas gene;

153 5) sequences in the neighbouring genes (Cas or others) when these sequences were found 154 besides an IS6110 sequence during reconstruction -see below- (for more details; see (Guyeux, 155 et al., 2019a)).

156 An extended version of these sequences of interest is presented in Supplementary file 1. 


\section{Locus reconstruction}

An automated contig building method based on De Bruijn approach was set up to reconstruct large fragments of the CRISPR. CRISPR with IS6110 insertion could not directly be reconstructed as no read can overlap the full IS6110 sequence (1,355 bp in length). Another reason for non-resolution of contigs is the existence of duplications: they lead to bifurcations in the de Bruijn graph. A specific search for duplications was included looking for patterns of the form $\mathrm{sp}(\mathrm{l}) * \mathrm{DRX}^{*} \mathrm{sp}(\mathrm{m})$, where $1 \geq \mathrm{m}$ (for more details; see (Guyeux, et al., 2019a)).

To facilitate the contigs concatenation, sequences were simplified by replacing each subsequence of interest by its name according to the catalogue described above. Final reconstruction taking into account IS6110 insertions was performed manually. In some samples, contig reconstruction was confirmed by retrieving the identity of the spacer downstream the last spacer of a duplication. When one side of the CRISPR could not be automatically recovered for instance due to an IS6110 insertion with a single end found in the catalog of CRISPR locus sequences, a stepwise manual search for the neighbouring sequences was performed until recovery of the other IS6110 end. The 60nt sequence found nearby was labelled according to the gene it belongs to and its position, and it was added to the catalog of sequences of interest.

\section{Results}

\section{Exhaustive catalog of spacers in M. tuberculosis complex stricto sensu}

We set up a method to identify not only variant of known spacers but also unknown spacers from M. tuberculosis CRISPR locus. Surprisingly, despite having explored more than 1,000 sequencing data (Guyeux, et al., 2019a), we found no new spacers as compared to the 68 described previously for M. tuberculosis sensu stricto (excluding M. canettii or the new L0 and L8 lineages) (van Embden, et al., 2000). The only new spacers that could be identified were found in $M$. canettii (data not shown). To identify whether this absence of new spacers could be due to a lack of sampling, we counted the cumulative number of spacers from the subset of isolates further described in this study upon 15 independent random samplings (Figure 1). We found that the 68 known spacers were all sampled after having examined from 
3 to 25 isolates. Our sampling was therefore one order of magnitude above the one that seems necessary to be exhaustive.

\section{Global structure of M. tuberculosis CRISPR}

We reconstructed the whole CRISPR loci for 198 clinical isolates representative of all $M$. tuberculosis diversity excluding M. canettii. CRISPR is almost always preceded by a complete set of cas genes, was followed by $R v 2813$, circumvented by one Direct Repeat sequence, DR0, at each of its border as can be seen for archetypal isolates from each Lineage (Figure 2). External DR0s are bordered by specific sequences, one of 48nt in length at the beginning of the locus, after Cas2, one of 148nt at the end of the locus, before Rv2813 (Supplementary file 1). These sequences are found in all isolates except in the case of large deletions (Supplementary file 2[IS6110 sheet]). Most of the times, the CRISPR-Cas locus includes one IS6110 copy as in the first isolate presented in Figure 2 belonging to L1.1.1.6 (ERR751749), but it can go up to three copies or down to zero (Supplementary file 2[IS6110 sheet]). No other type of insertion sequence was ever discovered inside the region (data not shown).

The spacer sequences as well as those of the DR are always found in the same direction. Their order of succession is usually the expected one (the order of natural integers) although, as described below, various particular situations arise, for instance in case of duplications (Supplementary file 3). Duplications are identified not only by the order of successive spacers, but also by the relatively higher number of reads corresponding to the duplicated spacers. For instance, in an isolate belonging to L1.1.1.8 (ERR718201), while most spacers were found on an average of 27 reads, spacers 14 to 21 are found in 56 reads on average, which is approximately twice as much (Figure 3). A notable exception in this isolate is spacer 16 that is found in only 31 reads. This however matches the fact that spacer 15 is half of the time followed by spacer 16 and the other half by spacer 17: in one of the two spacer 14-spacer 21 region, DVR16 has been deleted (Figure 3).

Duplications occur in tandem most of the time. For instance, a second DVR21 is found after its normal copy in L2 isolates such as ERR234109, and an additional tandem DVR1-DVR2 is found downstream the standard pair in M. bovis ERR5022499 (Figure 2). Other examples include DVR32 in ERR234197 (L1.1.3.1), DVR39 in ERR234248 (L2.1). This can be seen directly in the Illumina sequences, for instance for ERR234248, where many reads contain the 
221 end of 39, followed by a DR0, followed by the beginning of another 39, which has no chance

222 of happening, in such a repeated way, by chance due to random reading noise. A notable

223 exception to the natural order of succession of spacer is the case of the spacer 35, which can

224 be found in the following two places: between 34 and 36 on the one hand, and after 41 on the

225 other hand (Figure 2, Supplementary file 4). Consequently, in most cases, although this is

226 not the case of H37Rv and related isolates, there are two copies of 35.

227 Another important and widely representative characteristic of MTC CRISPR locus is the 228 presence of the IS6110 copy referenced in (Kamerbeek, et al., 1997) and that shares the same 229 orientation than the CRISPR, i.e. corresponding to a IS6110c (Figure 2).

\section{Punctual variants in M. tuberculosis CRISPR}

232

233

234

235

236

237

238

239

240

241

242

243

244

Regarding intra-spacer diversity, we identified 20 spacers that harbored at least two variants, and concerned $48(24 \%)$ out of the 198 isolates explored (Supplementary file 2[spacer sheet]). These variants consisted mainly of SNP, although a deletion was found in spacer 24 in another dataset (genome ERR702419, lineage 5, data not shown). Interestingly, some of these variants are characteristic of specific lineages. For instance, a variant of spacer 38 is found in all isolates of lineage L1.1.1, one mutation is found in spacer 4 in all L6 isolates to which an additional one sometimes adds resulting in two possible variants. Two variants of spacer 6 characterize the endemic Abyssinian L7 isolates (Figure 2, Supplementary file 5). The frequency of spacer variants in L2-L3-L4-L7 was relatively low (6 independent variants detected in 107 isolates, 5\%), as compared to L1 lineage (11 independent variants out of a selection of 55 isolates, $\sim 20 \%$ ) and lineage gathering animal isolates and L5 and L6 (7 independent variants for 34 isolates, $\sim 20 \%$ ).

Between two spacers, we have most of the time the DR0 sequence referenced in (van Embden, et al., 2000). However, this rule is incomplete and not general. Punctual variants were identified. First of all, between spacers 30 and 31, there is always, whatever the lineage, a sequence that we coined DR2 and that has one punctual mutation as compared to DR0 (see sequence in Supplemental file 1). Similarly, there is always a DR4 variant repeat between spacers 66 and 67, and again a DR5 variant between spacers 67 and 68. This is true for all lineages, with the notable exception of a sublineage of L6, which has yet the DR10 variant (Figure 2, Supplementary file 2[DR sheet]). Then, other types of variations were identified. For instance, between spacers 25 and 26, there are always only the last 24 nt of DR0 (a 
253

254

255

256

257

258

259

260

261

262

263

264

265

266

267

268

269

270

271

272

273

274

275

276

277

278

279

280

281

282

283

284

sequence we name DRb2). Around the central IS6110c, between spacers 34 and 35, the DR0 is split into two subsequences rDRa1 (upstream) and DRb1 (downstream). As expected due to IS6110 insertion characteristics, the concatenation of these two sequences is 3nt larger than DR0 since 3 additional cytosines are present at each end of the insertion (Gonzalo-Asensio, et al., 2018; Thierry, et al., 1990). Yet, in a L5.1 isolate (ERR702419) where IS6110c inserted downstream spacer 44, IS6110c is preceded by the first $35 \mathrm{nt}$ of DR0 and followed by its 6 last nt, so that the duplicated target was this time 5nt in length (data not shown).

Some variants are shared over several but not all lineages or sublineages. For instance, DR6 is found between spacers 64 and 65, in all genomes of lineages L2 to L4, and only in those; DR10 is found between spacers 67 and 68 in L6. Similarly, the DR1 variant is found between 14 and 15 only in Sublineage L1.1.1, and never in Sublineage L1.1.2, or in any other lineage. These findings are consistent with $M$. tuberculosis phylogeny and allow to infer that the mutation in L1.1.1 occurred shortly after separation from the rest of the other L1 sublineages.

Other punctual variants affect a single isolate (Supplementary file 2[spacer and DR sheets] for isolates affected, Supplementary file 1 for their sequence). Each time, the size of the DR is respected (no indel, only the single nucleotide polymorphism) except for one case where a longer DR was found (data not shown). Altogether, these variants occurred all over the locus with no clear preferential subregion (Supplementary file 6).

\section{Large scale variations and IS6110 copies}

Large scale variations included on one hand deletions and, on the other hand, duplications. It should be noted that, at this stage, no inversion has been detected in MTC CRISPR.

Large-scale deletions were observed throughout the lineages, such as the one characterizing L2.2/Beijing sublineage that covers parts of $\operatorname{csm} 4$ to an IS6110 just before spacer 46 (\#36 in the old nomenclature). As in the case of this specific deletion, many deletions were flanked by an IS6110 insertion: the deletion between spacer 33 and spacer 45 in L3 isolates ERR234109, and the deletion between spacer 11 and spacer 35 in L7 isolates ERR1971863 (Figure 2). To infer potential intermediates for these deletions, we searched for clinical isolates related to the one carrying deletions, and harbouring several IS6110 sequences. We found such evidence in Sublineage L4.1.2.1 (Haarlem sublineage). In this sublineage, a first set of isolates carry a 7 DVR- deletion adjacent to an IS6110 copy, namely between spacers 34 and the second copy of spacer 35 (for instance in ERR234259). A second set of clinical isolates (SRR5073877 and 
ERR552680) harbours two IS6110 copies, respectively the well-known one in the DR between spacers 34 and spacer 35, and another one in the DR between spacer 41 and the second spacer 35 (Figure 4). Interestingly, the borders of IS6110 insertion in ERR234259 corresponded well to the external borders of the two IS present in SRR5073877 and ERR552680. The left border consisted in the 17 first nt of DR0 (2nt less only than the rDRa1 in the classical position), and the right border was the exact same 33-last nucleotides of DR0 than the one found at the right of the second insertion in SRR5073877 and ERR552680. The CRISPR version with the two copies shares many features with that carrying the deletion, suggesting that it could correspond to its ancestral stage of evolution (Figure 4). The similar observation in L4.1.2.1 was made independently in a study performed in Hanoi (Maeda, et al., 2020).

These large scale deletions involved cas flanking genes in 23/198 (12\%) of isolates, with two different borders in L2 isolates, two others in L4 and a third one in L3. In contrast, a single case was observed that affected $R v 2813$ (Supplementary file 2[IS6110 sheet]). We further explored this asymmetry using SITVIT2 2019 database ( $\mathrm{n}=3852$ SITs): 290 SITs harbored a deletion of spacer \#1 (DVR2 in the new nomenclature) against 117 SITs with a deletion of spacer \#43 (DVR65 in the new nomenclature), i. e. three times more deletions on the cas genes side.

\section{Likely MRCA CRISPR of M. tuberculosis}

All variations we observed were concordant with the phylogeny of M. tuberculosis. We could thus infer the most likely structure of CRISPR locus of M. tuberculosis complex sensu stricto (without $M$. canettii), as well as its structure in all MRCA lineages. We found that global MRCA likely carried a full set of cas genes, a CRISPR with 69 spacers (the 68 spacers of different sequences + the repetition of spacer 35) interspersed mostly by DR0 except between spacers 25 and 26 (DRb2), spacers 30 and 31 (DR2), spacers 66 and 67 (DR4) and spacers 67 and 68 (DR5). An ancestral and central IS6110c was inferred to lie at the same place as the one occupied in H37Rv, i.e. between spacers 34 and 35 (Figure 4). A deletion of DVR 54 to 61 characterized MRCA of lineages 2, 3, 4 and 7, which is not documented in the classical form of the spoligotype as these spacers are not belonging to its set of 43 spacers. Other deletions corresponded to the ones found in spoligotype-43 format and used to define main sublineages. For instance, the deletion of spacers \#33-36 in the old nomenclature for L4/EuroAmerican lineage (previously referred to as T family) corresponds to the deletion of DVR43 to 50. Another example is the deletion of spacers \#29-32, presence of spacer \#33 and absence 
of spacer \#34 characteristic of Lineage 1 (previously referred to as EAI) (Filliol, et al., 2003) that corresponds to the deletion of DVR39 to 42, presence of DVR43 and absence of DVR44 (Figure 4). Only L2 MRCA did not carry the well-known signature of Beijing isolates as L2 includes not only the Beijing L2.2 sublineage but also the L2.1 proto-Beijing sublineage (Shitikov, et al., 2017). Interestingly, this ancestor harbors an IS6110 insertion in one cas gene (namely csm6) but not at the border of the classical Beijing deletion. It also lacks DVR16 and DVR17.

\section{Discussion}

Thanks to our new Sequence Reads Archive-based genomic analysis pipeline, we explored the $M$. tuberculosis CRISPR sequences diversity in 198 clinical isolates representative of the MTC excluding M. canettii, which deserve new specific studies (Supply, et al., 2013; van Soolingen, et al., 1997). These data show that M. tuberculosis CRISPR locus can contains at most 69 spacers ( $68+$ one duplication), is not prone to inversions, evolves by duplication and deletions through recombination between DR, but also and primarily through insertion/deletions implicating IS6110, by homologous recombination, and independently of lineage. We detail below the support for these different kinds of mutations and inferences that can be drown concerning the functionality of CRISPR-Cas locus.

\section{Evolutionary mechanisms of MTC CRISPR locus expansion}

Despite the absence of acquisition of new spacers, MTC CRISPR locus is of relative long size in many isolates (for instance, 4,589 nt between Rv2813 and Rv2816c/cas2 in H37Rv). This relates to its ability tocontinue to expand using mechanisms other than classical CRISPR adaptation.

A first mechanism of MTC CRISPR size expansion, when considered as the distance between its two borders, is the integration of IS6110 insertion sequences (1,355 bp). The most frequent insertion is found between spacers 34 and 35 as in H37Rv genome. Other IS6110 insertions were found along the whole MTC CRISPR locus, with up to two insertions in the CRISPR locus and three when considering the whole CRISPR-Cas locus. Other similar IS Sequences right next to or farther away, might be responsible for other homologous recombination mechanisms involving CRISPR.

The second CRISPR expansion mechanism identified in this overall review concerns duplications of DVR (DR + spacer). These duplications are of two main types. First of all, duplications can concern single DVR and place in tandem which was observed in 11 
350 independent cases throughout our 198 samples. This type of tandem duplication concerns also

351 several adjacent DVRs such as DVR1-2 in $M$. bovis or DVR14-15-16-17-18-19-20 in

352

353

354

355

356

357

358

359

360

361

362

363

364

365

366

367

368

369

370

371

372

373

374

375

376

377

378

379

380

381

382

L1.1.1.7. Such multiple DVR duplications were observed 5 times in our sample, so that in total 16 independent events of tandem duplications were observed. The second type of duplications concerns DVR that are far away from their original position, a type we call "rearrangement duplications". This first concerns DVR35 located between DVR41 and DVR42 as already mentioned above and supposedly in MTC MRCA CRISPR. Other examples include a second copy of DVR3 found between DVR12 and 13 found in ERR036187 (L4.3.4.1), while in ERR234197 (L1.1.3.1), there is an additional copy of DVR38 between DVR55 and 56. In one instance, this concerned several adjacent DVRs: a second copy of DVR50-51-52-53 is found between DVR3 and 4 in ERR2245409 (L3.1.1). Altogether, this made a total of 4 independent rearrangement-duplications. The fact that rearrangement duplications are less common than standard duplications suggests that they occur less frequently and/or that they are less stable. If the stability of rearrangement duplications was low, there should be several cases of deletions between the two copies of DVR35 as they were likely already present in MTC MRCA. Yet, we observed no case where a deletion concerned solely the DVR between these two copies.

Overall, the proportion of genomes containing either several copies of IS6110 or a duplication of one of the forms listed above is important, showing that MTC CRISPR is much more variable than what could be derived from a standard 43 spacers spoligotyping analysis. This is true not only for the in vitro but also for the in Silico-based acquisition of the spoligotype, as the blast procedure used in the current analytic tools (Spolpred, SpoTyping) only provides information on the presence or absence of a given spacer: there is nothing quantitative or location-related in these approaches (Coll, et al., 2012; Xia, et al., 2016). Hence, on one hand, the representation of the CRISPR locus through a simple barcode of presence/absence of individual spacers hide these quantitative and localization information, wheras on another hand, a more extensive description of the CRISPR locus including duplications, insertions, point mutations, provides useful information to classify and/or cluster clinical isolates. Such an information is advantageously correlated with the current SNPs based taxonomical system of MTC genomes and enhance our understanding of isolates evolution (Coll, et al., 2014; Palittapongarnpim, et al., 2018; Shitikov, et al., 2017; Stucki, et al., 2016).

Combined Mechanism of CRISPR locus reduction: how does IS6110 contributes to the evolution of CRISPR locus in MTC? 
383 In addition to the undeniable expansion mechanisms mentioned above, CRISPR reduction 384 mechanisms also coexist, which -to some extent- explain some of the spacer block deletions in MTC spoligotypes.

The first potential mechanism is the simple loss of spacer, for instance by recombination between two adjacent DRs. For instance, clinical isolate ERR1203071 of L4.8 lacks spacer 1. In place, it harbors a one nucleotide variant of the beginning sequence, a DR0 and spacer 2. The principle of parsimony here tends to suggest that a recombination between the DR0 bordering spacer 1 led to this genotype. The same kind of recombination seems to occur on slightly higher number of DVR such as the DVR54-DVR61 deletion typical of L2-3-4-7. Recombination between perfect DR would be favored compared to mutated DR.

We can know confidently argue that the second highly frequent mechanism, that is at play for the largest suppressions of consecutive spacers, is an IS-linked three steps mechanism: (1) insertion or prior presence of a first copy of IS6110 (for instance that after spacer 34), (2) insertion of a second IS6110 copy at another location (e.g. in csm6 in the ancestor of L2, also seen in SRR1710060, see Supplementary file 2), and (3) recombination between the two IS6110 copies. This IS-mediated mechanism, that has been described in previous studies is a general mechanism, i.e. it happens independently of lineage and is the responsible of IS6110 convergence of IS copy numbers (Roychowdhury, et al., 2015). The final result is the change from $x$ to $x$-1 copies of IS6110, with the loss of all spacers between the two copies. This mechanism can be observed independently of lineages, for example, in lineage 4, in Haarlem (4.1.2.1): L4 ancestor has a single copy of IS between 34 and 35, then a second copy occurred in the ancestor of Haarlem L4.1.2 isolates as seen in ERR552680, between 41 and 35, and finally a deletion occurred leading to the loss of spacers 35 to 41 for some isolates such as ERR234259. It therefore seems reasonable to think that after the insertion after spacer 41, this copy of IS6110 has recombined with the one upstream of spacer 35. This mechanism is also at work elsewhere in the Haarlem isolates between $\operatorname{csm} 5$ and spacer 34 and between $\operatorname{csm} 5$ and spacer 41 (Supplementary file 2).

IS6110 insertions can take place in spacers or in DR and it is not necessary for an IS to be in a

411 DR to be able to recombine. For instance, in many L4.3 (LAM) clinical isolates where spacers 41231 to 34 (\#21-\#24) are missing, the successive sequences of interest are: the beginning of spacer 31 (\#21), an IS6110c, DRb1 and spacer 35. The last three sequences of interest are 414 found in the exact same order in undeleted isolates such as H37rv. This suggests that an 415 IS6110 copy was first inserted at the end of spacer 31, and that it later recombined with the 
416 one located between spacers 34 and 35. This recombination did not modify the flanking 417 sequences.

418 The orientation of the two IS6110 copies that recombined cannot always be derived due to the 419 lack of the ancestral versions. Still in several cases, we could identify isolates related to the 420 deleted ones, that carry the two IS6110 flanking the future deletion. This is true for the 421 IS6110 insertions having led to the deletion described in Figure 4. In that case, both 422 insertions were in the reverse sense as compared to H37Rv orientation and can be called 423 IS6110c. In another case, the isolate with two IS6110 insertions is SRR5073887 (L4.4.1): it 424 carries not only the standard IS6110c insertion between spacers 34 and 35 but also an IS6110 425 insertion in the sense direction at the $439^{\text {th }}$ nt of $\operatorname{csm6}$. The deletion in ERR2653229 (also 426 L4.4.1) flanked by the beginning of csm6 and DRb1 and spacer 35 with a sense IS6110 427 sequence in its middle (Supplementary file 2 [IS6110 sheet]) likely occurred through the 428 recombination of these two IS although they lie in opposite orientations. This phenomenon 429 was recently observed in several cases of IS6110 mediated deletions in L2 (Shitikov, et al., 430 2019).

\section{Variants and problems in spoligotyping}

433 How does the sequence diversity impact spoligotyping data? When performed in vitro, 434 spoligotyping consists first in the amplification of the CRISPR locus using primers facing the 435 outside of DR region, referred to as DRa and DRb, and second in the hybridization to probes 436 attached at a specific position on a membrane or another support. CRISPR sequences variants may reduce the efficiency of the process, whether at the amplification or at the hybridization step. The presence of intermediate signals in spoligotyping or discrepant results between in Silico and in Vitro-based spoligotypes has been documented by several authors (Abadia, et al., 2011; Meehan, et al., 2018). We looked for intermediate signals corresponding to variants. In the case of L6 clinical isolates that carry a variant of spacer 4 (spacer 3 in spoligo-43 nomenclature), we found no evidence of such report in the literature and in our own data (data 443 not shown). The same was true for spacer 38 (spacer 28 in spoligo-43 nomenclature) found in 444 L1.1.1 clinical isolates even if the mutation is relatively central in the probe (Supplementary file 5). 


\section{Asymmetric variations affecting of MTC CRISPR-Cas locus}

448 As described above, we identified punctual nucleotide mutations, duplications, IS insertions 449 and deletions along CRISPR-Cas locus. CRISPR are oriented loci that acquire new spacers at 450 the 5' end relative to their transcription direction (Barrangou, et al., 2007; Makarova, et al., 451 2018). It may therefore be expected that variations do not affect symmetrically this locus. To 452 explore and understand the consequences of this possibility, it is important to identify the 453 orientation of the CRISPR locus in question. Using RNAseq data on H37Rv, Wei et al. 454 showed that transcription occurs from spacer 1 towards spacer 68 (Wei, et al., 2019). We 455 independently confirmed this observation by the exploration of independent RNAseq data 456 from (Ignatov, et al., 2015; Rodriguez, et al., 2014) (Refregier et al. unpublished results). The 457 orientation presented in this study is thus the functional one. According to classical CRISPR 458 expansion mechanism, the introduction of new spacers occurs at the 5' end of the locus, so that the most ancient DVR lies at its 3' end.

460 In contradiction with the remarkable feature that most ancient DR carry mutations in all 461 isolates, no subregion exhibited a significantly higher punctual mutation rate (Supplementary

462 file 6). The fact that the most ancient part of CRISPR locus does not carry a significantly higher number of punctual mutations as compared to parts that are more recent (spacer block deletions), may suggest that the time during which the locus expanded from spacer 68 to spacer 1 may be negligible as compared to the time between MTC MRCA and present, or that the CRISPR locus was transferred by lateral gene transfer in one single block from another environmental organism. Alternatively, the time of CRISPR locus expansion could have been quite long, however the pace of CRISPR locus SNPs mutations acquisition was very slow because of an extremely slow pace of MTC transmission. Demography and genetic drift could have been much more important for MTC evolution than selection in human populations (Pepperell, et al., 2010). Yet, the presence of mutations in several DR at the 3' end of the locus could also play a role in its stability.

In contrast, we detected an asymmetry concerning the loss of flanking sequences: it was apparently more frequent to have a loss of the beginning sequences of CRISPR, on the side of the cas genes (several independent isolates from L2 and from L4) than to have a loss of the ending sequences, i.e. on the side of $R v 2813$. All deletions implicating flanking sequences were bordered by an IS6110 sequence. Altogether, the asymmetry in deletion suggests either a more crucial role of the end of the CRISPR i.e. of gene Rv2813 and/or its neighbors, or asymmetric mechanisms favoring deletion on the cas gene side. This second possibility 
relates to IS6110 insertion frequency as IS are always involved in large deletions. Saying that IS6110 insertions are more likely on the cas gene side suggests either their lower impact on bacterial fitness, or a DNA superstructure that would favor IS insertions. Other IS exist in the genome that could also insert in a favorable region. Their presence in CRISPR region would be a sign that it is an integration hot spot. However, our script was designed to look only for insertion in cas gene that also lead to a deletion in the CRISPR in at least one of the explored sample. IS other than IS6110 cannot lead to any deletion. Nevertheless, even if our script may have overlooked non-IS6110 insertions, we did not encounter it in around 500 randomly sampled genomes. The question of cas gene locus being an integration hotspot of IS sequences needs other studies to be completely solved.

\section{Functionality of MTC CRISPR-Cas locus}

CRISPR-Cas loci are involved in two mechanisms: 1) adaptation by the integration of new spacers, usually taken from foreign DNA, at the 5' end of CRISPR with the help of Cas1 and Cas2 proteins, and 2) immunity by the transcription of CRISPR locus, processing with the help of Cas6 protein in the case of type III-A CRISPRs, and degradation of DNA and/or RNA carrying protospacers, with the help of the crRNP (CRISPR RiboNucleoProtein complex), a complex involving the crRNA and other Cas proteins. By exploring the diversity of many genomes at the CRISPR locus, we are able to infer the effectivity of adaptation processes. Regarding immunity, we can only state whether the necessary genes are present or not.

In the whole M. tuberculosis complex sensu stricto, we could find only the 68 spacers already present in the MRCA (van Embden, et al., 2000). We found no evidence that a single clinical isolate has acquired a new spacer in the course of MTC evolution. This seems particularly surprising as most currently spreading isolates apart those from L2 still carry the full set of Cas genes including Cas 1 and Cas 2 involved in CRISPR adaptation in other type III-A systems. This could be due to a mutation in M. tuberculosis ancestor that has abolished Cas 1 and/or Cas 2 functionality in the ancestor. Another reason could be that MTC, given its intracellular life-style, does simply not have the chance anymore to encounter foreign DNA such as phages or plasmids. These two phenomena could also be linked: a loss of functionality of Cas1 and Cas2 in the MRCA of all MTC could have fostered an adaptative change in lifestyle of the bacterium, i.e. from an environmental extracellular to a host-specialized intracellular life-style. Such an hypothesis could be supported by the evolution of the CRISPR 
512 locus of Vibrio cholerae, with observations that the recent pandemic strains have lost their

513 ancestral CRISPR locus (Weill, et al., 2017) and (FX Weill, personal communication). Hence, 514 the functionality of Cas 1 and Cas 2 of MTC remains to be explored.

515 Regarding immunity, this study only focused on the full presence or absence of cas genes 516 without exploring in detail SNP variations. As stated previously, 23/198 (12\%) lacked at least 517 part of the cas genes. Among these yet, all isolates still carried the cas6, cas 10/csm1, csm2, 518 and $\operatorname{csm} 3$ genes. This observation matches that made previously on CRISPR clinical isolates 519 (Freidlin, et al., 2017). Cas6 protein is involved in pre-crRNA processing. Cas10/Csm1 and $520 \mathrm{Csm} 3$ are the enzymes responsible for the catalytic activity of the crRNP (Kazlauskiene, et al., 521 2017; Samai, et al., 2015). Hence, regarding immunity, even if the spatial structure of the crRNP may be impaired by the absence of $\operatorname{csm} 4$ and/or $\operatorname{csm} 5$ in some isolates, it could remain possible that immunity occurs in all MTC isolates through the consecutive actions of Cas 6 to process pre-crRNA and of Cas10/Csm1 and Csm3 to degrade DNA and/or RNA. The fact that none of the spacer is conserved in all isolates implies that, if immunity occurs, it does not always target the same DNA and/or RNA sequences.

Global Implication of CRISPR diversity for the understanding of MTC clinical isolates evolution

In MTC, the CRISPR locus is a likely witness of a previous yet unknown evolutionary history of phage DNA invaders defense, whereas IS6110 is a specific MTC element that belongs to the IS3 family that, through transposition, also plays a permanent role in shaping MTC genomes (Thabet and Souissi, 2017). The link between the two in evolutionary genomics remains poorly investigated until now. MTC genome actually contains a lot of other IS and transposases (88 genes retrieved in mycobrowser, (https://mycobrowser.epfl.ch/) such as IS1081, IS1533, IS1547, IS1560), but IS6110 is the one with the largest number of copies in most isolates and especially in the reference isolate H37Rv (Cole, et al., 1998). IS1547 was previously shown to play a role in MTC evolution however it remains poorly investigated 539 (Fang, et al., 1999). IS6110-RFLP was the golden standard to define epidemiological clusters 540 at the end of the nineties and stayed so during around 20 years, until it was replaced by 541 MIRU-VNTR ${ }^{1}$ and more recently by Whole-Genome-Sequencing (Schurch, et al., 2010; 542 Supply, et al., 2006; van Embden, et al., 1993; van Soolingen, et al., 2007) (for a recent

\footnotetext{
${ }^{1}$ Mycobacterial Interspersed Repetitive Units-Variable Number of Tandem Repeats Typing
} 
543 review on evolution of TB molecular epidemiological methods, see also (Garcia De Viedma 544 and Perez-Lago, 2018)). Previous results on IS6110 insertion sites have shown that 545 independent IS6110 copy acquisition through transposition into hot-spots was a common 546 mechanism explaining convergence in IS6110 copy number in some of the MTBC 547 sublineages (Dale, et al., 2003; Roychowdhury, et al., 2015). A recent paper on the micro- and 548 macro-evolution of Lineage 2 of MTC in relation to IS6110 transposition also stress the 549 interest of such studies using WGS (Shitikov, et al., 2019). The role of the ipl (Insertion 550 Preference Locus) was also stressed long time ago and showed consequences on the CRISPR 551 locus (Fang, et al., 1999; Fang, et al., 1999; Fang and Forbes, 1997), however no generalized 552 observations on IS-CRISPR genomics dynamics had been done so far before this study.

\section{Conclusions}

555 Our study, by providing an in-depth reconstruction of the CRISPR locus of MTC using short 556 reads on around 200 genomes, in combination with IS6110, improves our knowledge on the 557 structure of the CRISPR locus and sheds new light on the general evolutionary mechanisms 558 acting on MTC genomes through a first yet quantitatively limited analysis that combines 559 CRISPR-IS combined evolutionary dynamics. By unveiling an unexpected genetic diversity 560 of the CRISPR Locus on MTC, our study opens the way to new in-depth congruence analysis 561 between SNP-based and repetitive sequence based MTC phylogenies. Such deeper knowledge 562 on the natural history of tuberculosis will help us deciphering the most important key 563 evolutionary events that shaped today's global and local MTC genomes population structure.

\section{Declarations}


571 The authors declare no competing interest

572

573

574

575

576

577

578

579

580

581

582

583

584

585

586

587

588

589

590

591

592

593

594

595

596

\section{Funding}

This study was funded by CNRS (Centre National de la Recherche Scientifique), The University of Paris-Saclay and the University of Bourgogne Franche-Comté through recurrent research support to the research teams.

\section{Authors' contributions}

CG, GR, CS conceived the study. CG developed the pipeline, GC,GR,CS chose the genomes to be analyzed, GR and CG analyzed results helped by CS; GR, CS and CG wrote the manuscript, GR drew the Figures and built the Supplementary Tables;

\section{Acknowledgements}

Laura Morel, Valentin Pohyer, Matthieu Petrou, three previous undergraduates students who contribute to the start of the MTC CRISPR genome project are warmly acknowledged

\section{Data and Material availability}

All genomic data used were extracted from Public genome databases (NCBI or ENA archives). Computer Program specifically developed in this paper will be made freelu available upon request to Christophe Guyeux (christophe.guyeux @univ-fcomte.fr).

\section{References}

Abadia, E., et al. The use of microbead-based spoligotyping for Mycobacterium tuberculosis complex to evaluate the quality of the conventional method: providing guidelines for Quality Assurance when working on membranes. BMC Infect Dis 2011;11:110.

Barrangou, R., et al. CRISPR provides acquired resistance against viruses in prokaryotes. Science 2007;315(5819):1709-1712.

Blouin, Y., et al. Significance of the Identification in the Horn of Africa of an Exceptionally Deep Branching Mycobacterium tuberculosis Clade. PLoS One 2012;7(12):e52841. 
597 Brudey, K., et al. Mycobacterium tuberculosis complex genetic diversity : mining the fourth 598 international spoligotyping database (SpolDB4) for classification, Population Genetics, and 599 Epidemiology. BMC Microbiol. 2006;6(6):23.

600 Cole, S.T., et al. Deciphering the biology of Mycobacterium tuberculosis from the complete genome 601 sequence. Nature 1998;393(6685):537-544.

602 Coll, F., et al. SpolPred: rapid and accurate prediction of Mycobacterium tuberculosis spoligotypes 603 from short genomic sequences. Bioinformatics 2012;28(22):2991-2993.

604 Coll, F., et al. A robust SNP barcode for typing Mycobacterium tuberculosis complex strains. Nature 605 communications 2014;5:4812.

606 Coll, F., et al. PolyTB: A genomic variation map for Mycobacterium tuberculosis. Tuberculosis (Edinb) 607 2014;94(3):346-54(3):346-354.

608 Comas, l., et al. Genotyping of genetically monomorphic bacteria: DNA sequencing in Mycobacterium tuberculosis highlights the limitations of current methodologies. PLoS One 2009;4(11):e7815. Consortium, C., et al. Prediction of Susceptibility to First-Line Tuberculosis Drugs by DNA Sequencing. N Engl J Med 2018;379(15):1403-1415. Couvin, D., et al. CRISPRCasFinder, an update of CRISRFinder, includes a portable version, enhanced performance and integrates search for Cas proteins. Nucleic Acids Res 2018. Couvin, D., et al. Macro-geographical specificities of the prevailing tuberculosis epidemic as seen through SITVIT2, an updated version of the Mycobacterium tuberculosis genotyping database. Infect Genet Evol 2018. Dale, J.W., et al. Evolutionary relationships amongst isolates of Mycobacterium tuberculosis with few copies of IS6110. J. Bacteriol. 2003;185(8):2555-2562. Fabre, L., et al. CRISPR typing and subtyping for improved laboratory surveillance of Salmonella infections. PLoS One 2012;7(5):e36995.

Fang, Z., et al. IS6110-mediated deletions of wild-type chromosomes of Mycobacterium tuberculosis. Journal of bacteriology 1999;181(3):1014-1020.

Fang, Z., et al. Characterization of IS1547, a new member of the IS900 family in the Mycobacterium tuberculosis complex, and its association with IS6110. Journal of bacteriology 1999;181(3):10211024.

Fang, Z. and Forbes, K.J. A Mycobacterium tuberculosis IS6110 preferential locus (ipl) for insertion into the genome. J Clin Microbiol 1997;35(2):479-481.

Filliol, I., et al. Snapshot of moving and expanding clones of Mycobacterium tuberculosis and their global distribution assessed by spoligotyping in an international study. I Clin Microbiol 2003;41(5):1963-1970.

Freidlin, P.J., et al. Structure and variation of CRISPR and CRISPR-flanking regions in deleted-direct repeat region Mycobacterium tuberculosis complex strains. BMC genomics 2017;18(1):168.

Gagneux, S. Host-pathogen coevolution in human tuberculosis. Philosophical transactions of the Royal Society of London. Series B, Biological sciences 2012;367(1590):850-859.

Garcia De Viedma, D. and Perez-Lago, L. The Evolution of Genotyping Strategies To Detect, Analyze, and Control Transmission of Tuberculosis. Microbiology spectrum 2018;6(5).

Gonzalo-Asensio, J., et al. New insights into the transposition mechanisms of IS6110 and its dynamic distribution between Mycobacterium tuberculosis Complex lineages. PLoS genetics 2018;14(4):e1007282.

Grissa, I., et al. On-line resources for bacterial micro-evolution studies using MLVA or CRISPR typing. Biochimie 2008;90(4):660-668.

Grissa, I., Vergnaud, G. and Pourcel, C. The CRISPRdb database and tools to display CRISPRs and to generate dictionaries of spacers and repeats. BMC Bioinformatics 2007;8:172.

Grissa, I., Vergnaud, G. and Pourcel, C. CRISPRFinder: a web tool to identify clustered regularly interspaced short palindromic repeats. Nucleic Acids Res 2007;35(Web Server issue):W52-57.

Groenen, P.M., et al. Nature of DNA polymorphism in the direct repeat cluster of Mycobacterium tuberculosis; application for strain differentiation by a novel typing method. Molecular microbiology 1993;10(5):1057-1065. 
649 Guyeux, C., Sola, C. and Refrégier, G. Exhaustive reconstruction of the CRISPR locus in M. tuberculosis 650 complex using short reads Bioinformatics 2019a;submitted.

651 Hershberg, R., et al. High functional diversity in Mycobacterium tuberculosis driven by genetic drift 652 and human demography. PLoS biology 2008;6(12):e311.

653 Ignatov, D.V., et al. Dormant non-culturable Mycobacterium tuberculosis retains stable low654 abundant mRNA. BMC genomics 2015;16:954.

655 Ishino, Y., et al. Nucleotide sequence of the iap gene, responsible for alkaline phosphatase isozyme 656 conversion in Escherichia coli, and identification of the gene product. Journal of bacteriology 657 1987;169(12):5429-5433.

658 Jajou, R., et al. Epidemiological links between tuberculosis cases identified twice as efficiently by 659 whole genome sequencing than conventional molecular typing: A population-based study. PLoS One 660 2018;13(4):e0195413.

661 Jansen, R., et al. Identification of genes that are associated with DNA repeats in prokaryotes. 662 Molecular microbiology 2002;43(6):1565-1575.

663 Kamerbeek, J., et al. Simultaneous detection and strain differentiation of Mycobacterium tuberculosis for diagnosis and epidemiology. J Clin Microbiol 1997;35(4):907-914.

Kato-Maeda, M., et al. Strain classification of Mycobacterium tuberculosis: congruence between large sequence polymorphisms and spoligotypes. Int J Tuberc Lung Dis 2011;15(1):131-133.

Kazlauskiene, M., et al. A cyclic oligonucleotide signaling pathway in type III CRISPR-Cas systems. Science 2017;357(6351):605-609.

Maeda, S., et al. Genotyping of Mycobacterium tuberculosis spreading in Hanoi, Vietnam using conventional and whole genome sequencing methods. Infection Genetics Evolution 2020;78:104107.

Makarova, K.S., et al. An updated evolutionary classification of CRISPR-Cas systems. Nature reviews. Microbiology 2015;13(11):722-736.

Makarova, K.S., Wolf, Y.I. and Koonin, E.V. Classification and Nomenclature of CRISPR-Cas Systems: Where from Here? CRISPR J 2018;1(5):325-336.

Meehan, C.J., et al. The relationship between transmission time and clustering methods in Mycobacterium tuberculosis epidemiology. EBioMedicine 2018;37:410-416.

Mulholland, C.V., et al. Dispersal of Mycobacterium tuberculosis driven by historical european trade in the South Pacific. Frontiers in microbiology 2019;doi: 10.3389/fmicb.2019.02778.

Ngabonziza, J.C.S., et al. An ancestral lineage of the Mycobacterium tuberculosis complex discovered near the African Great Lakes, missing link between M. canettii and M. tuberculosis sensu stricto. In, European Society Microbiology Congress. Valencia; 2019.

682 Palittapongarnpim, P., et al. Evidence for Host-Bacterial Co-evolution via Genome Sequence Analysis 683 of 480 Thai Mycobacterium tuberculosis Lineage 1 Isolates. Scientific reports 2018;8(1):11597.

684 Pepperell, C., et al. Bacterial genetic signatures of human social phenomena among M. tuberculosis from an Aboriginal Canadian population. Molecular biology and evolution 2010;27(2):427-440.

Pourcel, C., Salvignol, G. and Vergnaud, G. CRISPR elements in Yersinia pestis acquire new repeats by preferential uptake of bacteriophage DNA, and provide additional tools for evolutionary studies. Microbiology 2005;151(Pt 3):653-663.

689 Rodriguez, J.G., et al. Global adaptation to a lipid environment triggers the dormancy-related 690 phenotype of Mycobacterium tuberculosis. mBio 2014;5(3):e01125-01114.

691 Roychowdhury, T., Mandal, S. and Bhattacharya, A. Analysis of IS6110 insertion sites provide a 692 glimpse into genome evolution of Mycobacterium tuberculosis. Scientific reports 2015;5:12567.

693 Samai, P., et al. Co-transcriptional DNA and RNA Cleavage during Type III CRISPR-Cas Immunity. Cell 694 2015;161(5):1164-1174.

695 Schurch, A.C., et al. High resolution typing by integration of genome sequencing data in a large 696 tuberculosis cluster. J Clin Microbiol 2010;48(9):3403-3406.

697 Schurch, A.C., et al. The tempo and mode of molecular evolution of Mycobacterium tuberculosis at 698 patient-to-patient scale. Infect Genet Evol 2010;10(1):108-114. 
699 Shitikov, E., et al. The role of IS6110 in micro- and macroevolution of Mycobacterium tuberculosis 700 lineage 2. Molecular phylogenetics and evolution 2019;139:106559.

701 Shitikov, E., et al. Evolutionary pathway analysis and unified classification of East Asian lineage of 702 Mycobacterium tuberculosis. Scientific reports 2017;7(1):9227.

703 Stucki, D., et al. Mycobacterium tuberculosis lineage 4 comprises globally distributed and 704 geographically restricted sublineages. Nature genetics 2016;48(12):1535-1543.

705 Supply, P., et al. Proposal for standardization of optimized mycobacterial interspersed repetitive unit706 variable-number tandem repeat typing of Mycobacterium tuberculosis. I Clin Microbiol 707 2006;44(12):4498-4510.

708 Supply, P., et al. Genomic analysis of smooth tubercle bacilli provides insights into ancestry and pathoadaptation of Mycobacterium tuberculosis. Nature genetics 2013;45(2):172-179.

Thabet, S. and Souissi, N. Transposition mechanism, molecular characterization and evolution of IS6110, the specific evolutionary marker of Mycobacterium tuberculosis complex. Mol Biol Rep 2017;44(1):25-34.

Thierry, D., et al. IS6110, an IS-like element of Mycobacterium tuberculosis complex. Nucleic. Acids. Res. 1990;18:188.

van Belkum, A., et al. short-sequence DNA repeats in prokaryotic genomes. MMBR 1998;62:275-293. van Embden, J.D., et al. Strain identification of Mycobacterium tuberculosis by DNA fingerprinting: recommendations for a standardized methodology. J Clin Microbiol 1993;31(2):406-409.

van Embden, J.D.A., et al. Genetic variation and evolutionary origin of the Direct repeat locus of Mycobacterium tuberculosis complex bacteria. J. Bacteriol. 2000;182:2393-2401.

van Soolingen, D., et al. A novel pathogenic taxon of the Mycobacterium tuberculosis complex, Canetti: characterization of an exceptional isolate from Africa. Int J Syst Bacteriol 1997;47(4):12361245.

van Soolingen, D., Kremer, K. and W.M., H.P. Molecular Epidemiology: Breakthrough Achievements and Future Prospects. In: Amadeo, editor, Tuberculosis 2007 : from basic sicence to patient care Amadeo; 2007. p. Chapter 9.

Wei, J., et al. The Mycobacterium tuberculosis CRISPR-Associated Cas1 Involves Persistence and Tolerance to Anti-Tubercular Drugs. Biomed Res Int 2019;2019:7861695.

Weill, F.X., et al. Genomic history of the seventh pandemic of cholera in Africa. Science 2017;358(6364):785-789.

Xia, E., Teo, Y.Y. and Ong, R.T. SpoTyping: fast and accurate in silico Mycobacterium spoligotyping from sequence reads. Genome medicine 2016;8(1):19. 


\section{$735 \quad$ Figure legends}

736 Figure 1 - Cumulative number of spacers along random sampling of our database.

737 Figure 2 - CRISPR-Cas locus reconstitution for one archetypal isolate of each lineage.

$738 \quad$ Notes common to fig. 2 and 3:

739 Arrows indicate genes. Diamonds indicate spacers. Boxes indicated Direct Repeats (DR).

740 Width of spacers are DR has been articially expanded for clarity. The pink empty box

741 highlights a duplicated spacer at an unexpected position (not in tandem).

742 Color codes for genes (arrows): light blue: cas genes involved in immunity (interference);

743 dark blue: cas genes involved in adaptation; green : IS6110 genes (transposase and

744 hypothetical protein); white: other neighbouring gene of unknown function.

745 The color of spacers was attributed randomly to facilitate visual exploration but spacers of the

746 same color have no link except if they carry the same number.

747 Direction of CRISPR-Cas locus is antisense as compared to $\mathrm{H} 37 \mathrm{Rv}$ genome orientation, so 748 that all cas genes are annotated with a c: cas6 is Rv2824c and cas2 is Rv2816c. Genes 749 forming the IS6110 sequence are sometimes in the sense and sometimes in the antisense 750 direction. Between spacers 34 and 35 as in H37Rv, there are in the antisense direction and 751 therefore are referred to as Rv2815c and Rv2814c.

752 Several DRs are truncated. Between spacers 34 and 35, IS6110c is preceded by a sequence 753 close to rDra, corresponding to the 19 first nt of DR0 (shown in light grey), and is followed 754 by a sequence close to Drb (referred to as DRb1) corresponding to the 20 last nt of DR0 755 (darker grey). These two sequences therefore share the CCC sequence in the middle of DR0. 756 They are also found around the IS6110c sequence of L7 isolates. A similar case is true in L5.1 757 ERR7022419 clinical isolates. Around the IS6110c copy in ERR234109 (L3), the preceding 758 spacer is slightly truncated (sp33, only its first $27 \mathrm{nt}$ ), and there are only the last 4 nucleotide 759 of the DR0 before the nest spacer (sp45).

760 When a DR0 borders a deletion, we chose to represent it in most of the cases at the beginning 761 of the deletion, although choosing the end of the deletion would have been equally relevant.

762 Mutated DR are indicated in black. They are not the same from one position to the other, but 763 variants at the same location are the same except for the DR between spacers 67 and 68 that 
764 harbors a second variant solely in L6 and is therefore indicated by a star (see Supplementary

765 file 3).

766 Figure 3 - Proof for spacers 14-20 duplication in isolate ERR718201. Reads number as a

767 function of spacer number is shown in blue. The number of the following spacer is shown in

768 red (crosses).

769 Figure 4 - CRISPR substructures of related isolates illustrating deletion by 770 recombination between IS6110 copies. ERR072087 with one single copy with all spacers in 771 the subregion of interest likely harbors the most ancestral structure. ERR552680 with two 772 copies and all spacers likely represents an intermediate state after a new IS6110 insertion. 773 ERR234259 with a single copy and loss of spacers likely emerged due to the recombination 774 between the two copies present in ERR552680.

Figure 5 - CRISPR-Cas locus likely structure of each lineage MRCA

777 The proposed structure was designed by a parsimonious approach based on the CRISPR structure of the 198 clinical isolates fully characterized in Supplementary file 3 (See also notes common with Fig. 2).

\section{Supplementary files}

784 Supplemental file 1 (doc) - Sequences of interest in CRISPR-Cas region of Mycobacterium tuberculosis complex.

Supplemental file 2 (tab) - CRISPR reconstructions highlighting 1) global structure and position of IS6110 insertions ['IS6110' sheet]; 2) spacer variants ['spacer' sheet]; 3) DR variants ['DR' sheet]; 4) Duplicated DVR ['Duplic' sheet].

Supplemental file 3 - Exploration of read numbers for the reconstruction and identification of duplications, the case of ERR718197.

791 Supplemental file 4 - Confirmation of sp35 presence after spacer 41 in two Sequence runs 792 from clinical isolatess belonging to L5 and L2 respectively 
793 Supplemental file 5 - Spacer 4, spacer 6 and spacer 38 variants in parallel with 43-spacers

794 spoligotyping probes

795 Supplemental file 6 - Cumulative punctual variant numbers 5DR variants + spacer variants)

796 in groups of 5 successive DVR from DVR1-5 to the last three DVR (DVR66-68)

797

798

799

800

801 
mulative spacer number

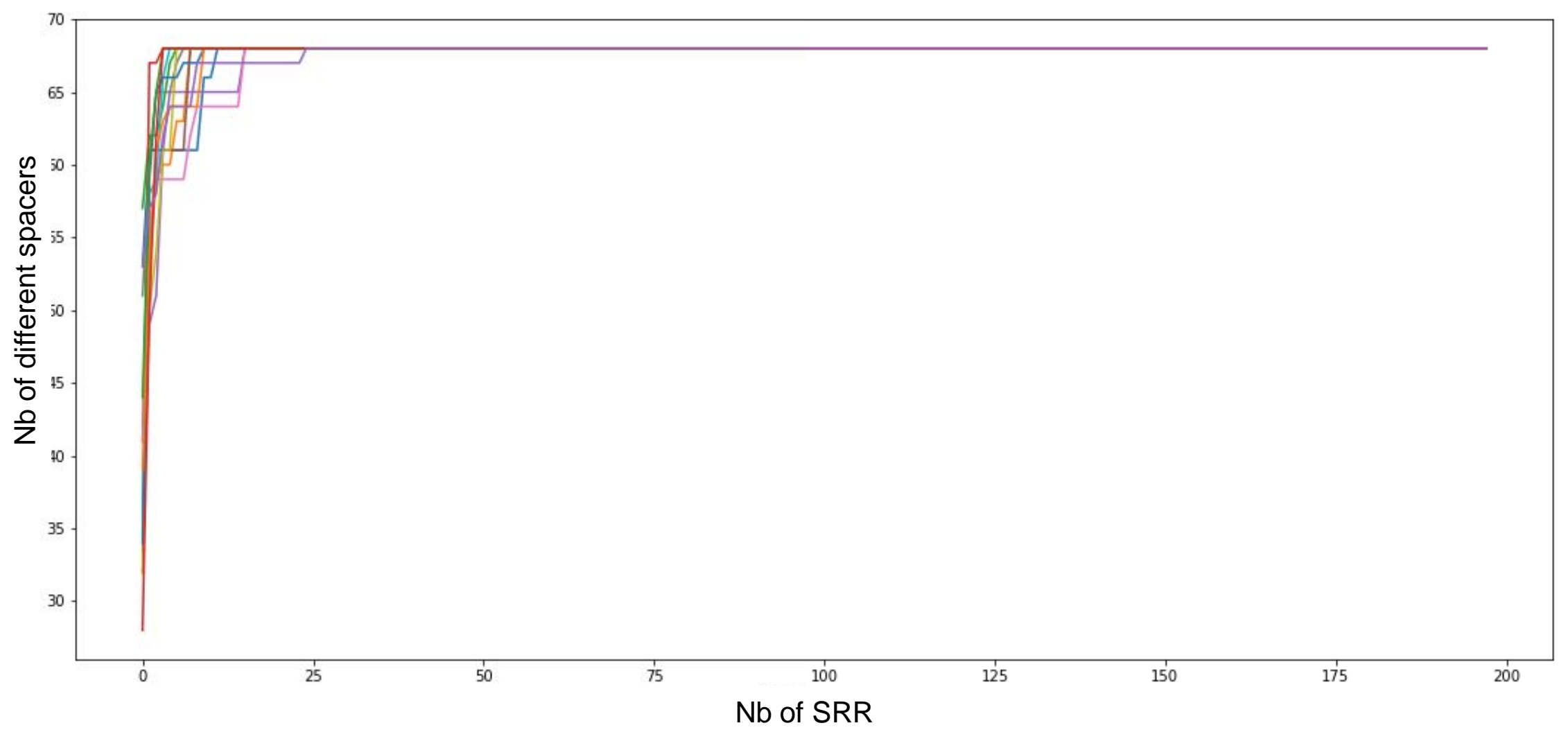


Fig2. Reconstitution of CRISPR-Cas locus for one archetypal isolate of each lineage

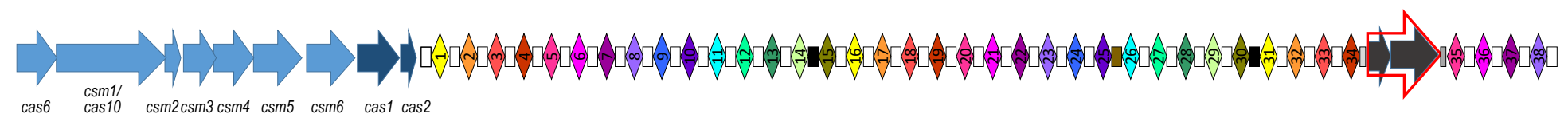

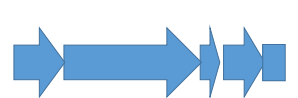

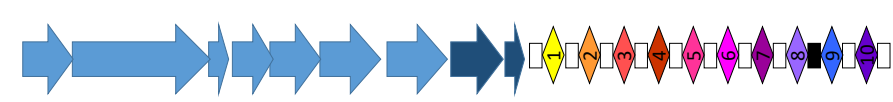

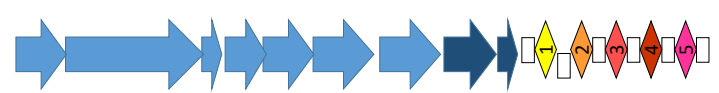

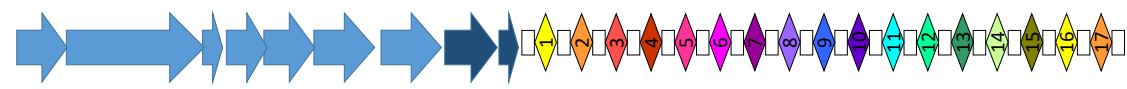

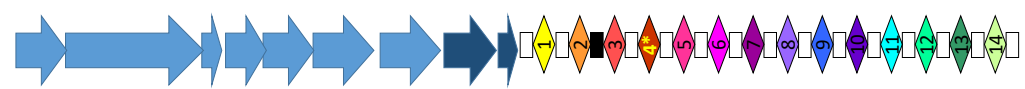

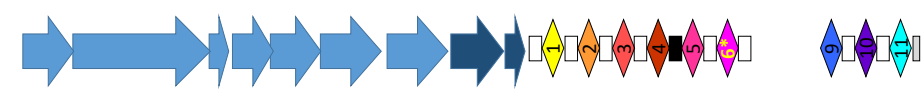

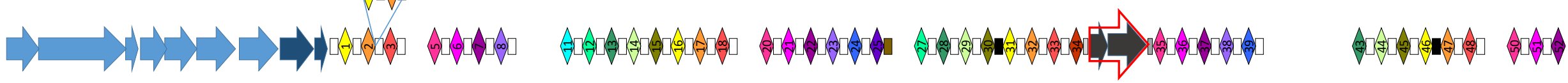

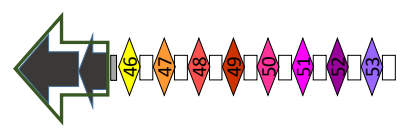

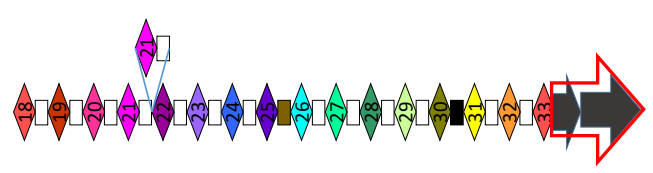

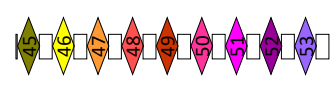

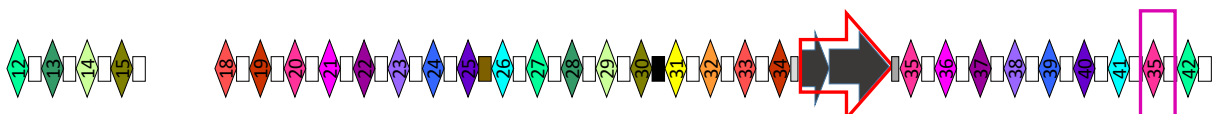

त)

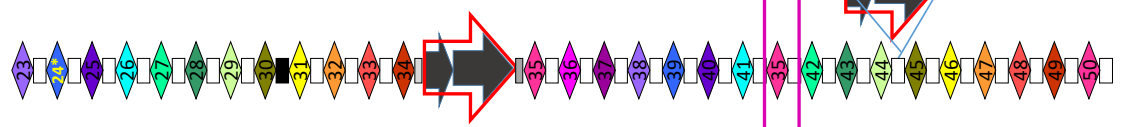

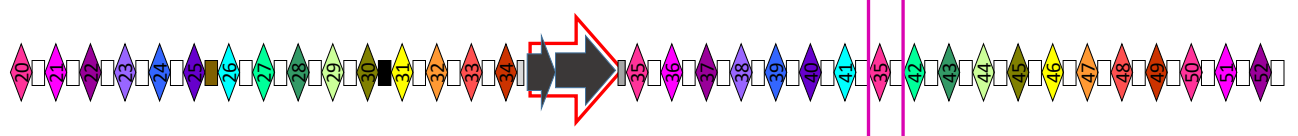

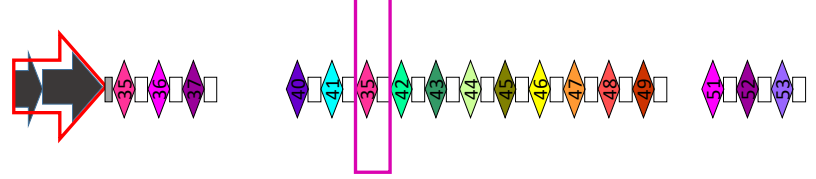

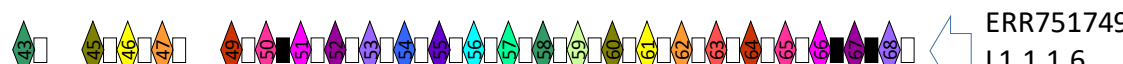

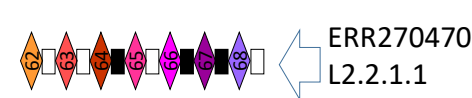

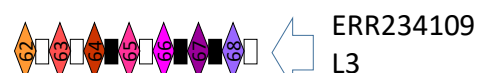

$\operatorname{Ar} A]$ 解

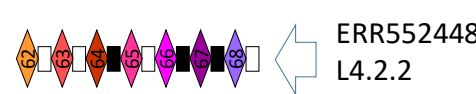

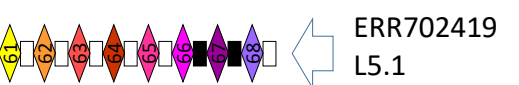

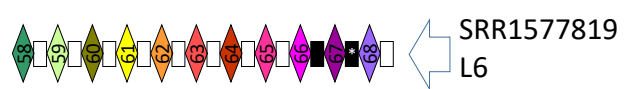

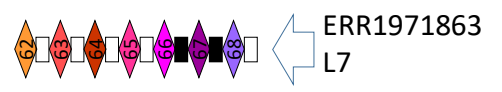


Fig. 3 - Proof for spacers 14-20 duplication in isolate ERR718201. Reads number as a function of spacer number are shown in blue. The number of the following spacer is shown in red (crosses).

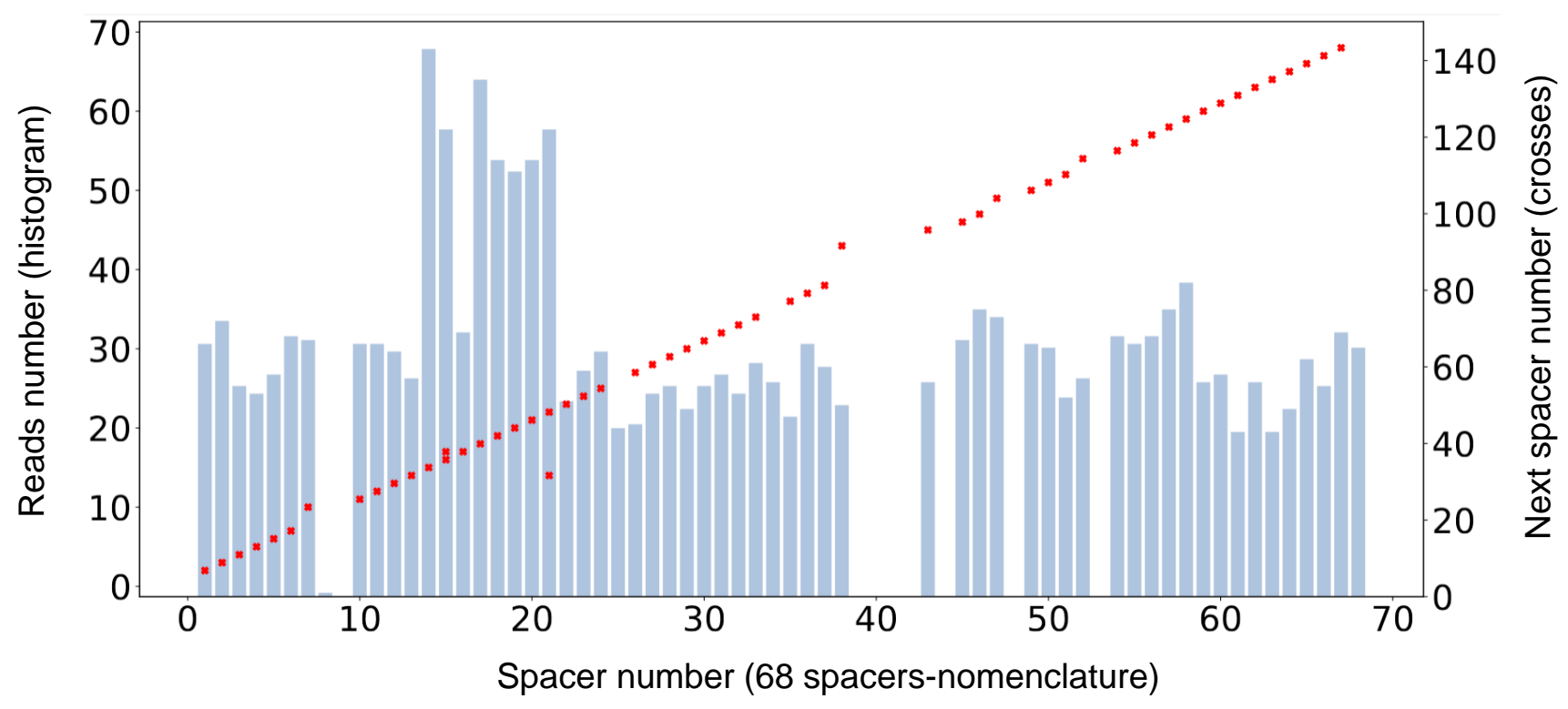


Figure 4 - CRISPR substructures of related isolates illustrating deletion by recombination between IS6110 copies. ERR072087 with one single copy with all spacers in the subregion of interest likely harbors the most ancestral structure. ERR552680 with two copies and all spacers likely represents an intermediate state after a new IS6110 insertion. ERR234259 with a single copy and loss of spacers likely emerged due to the recombination between the two copies present in ERR552680.

ERR072087, L4.1.1.3

ERR552680, L4.1.2.1

ERR234259, L4.1.2.1

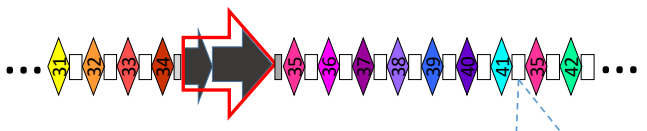
…

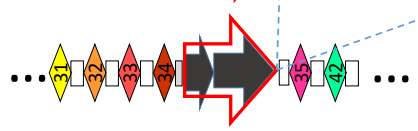


Figure 5 - CRISPR-Cas locus likely structure of each lineage MRCA

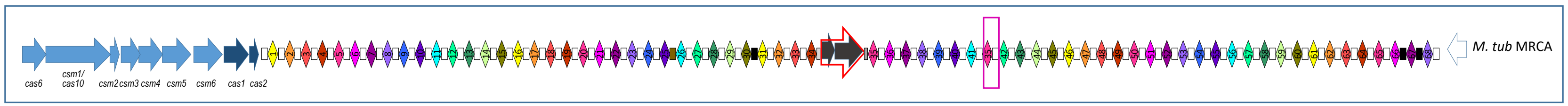

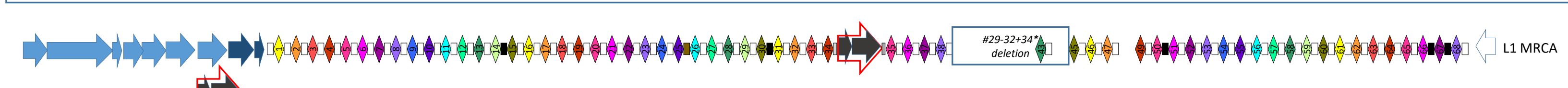
(2)

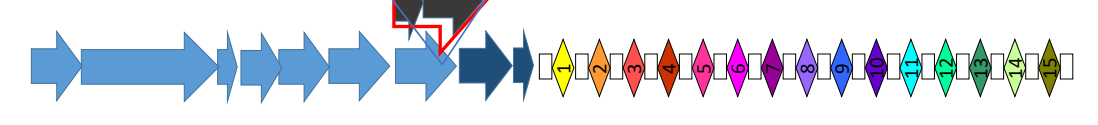

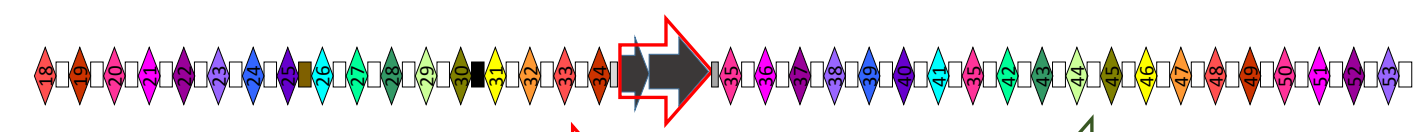

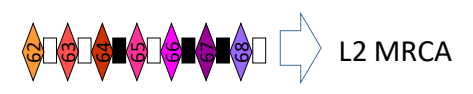

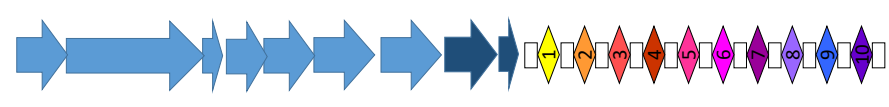

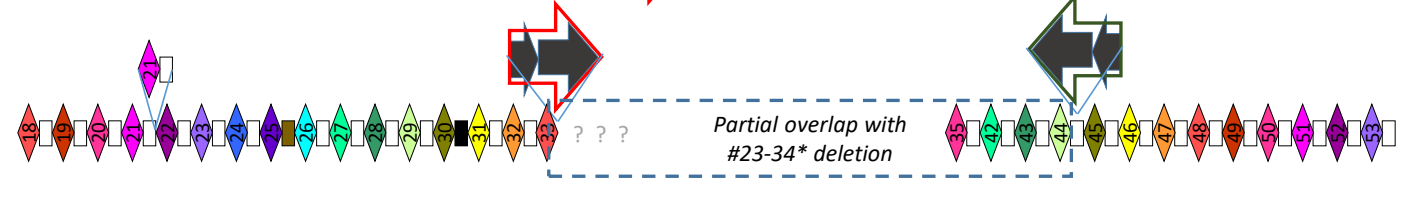

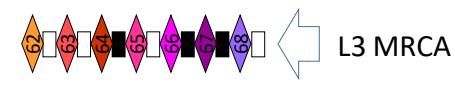

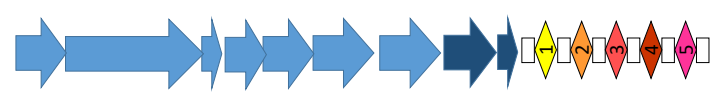

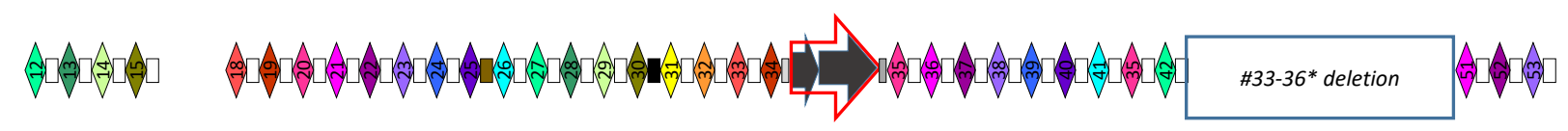

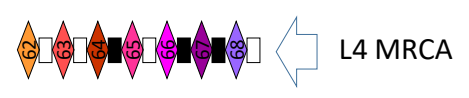

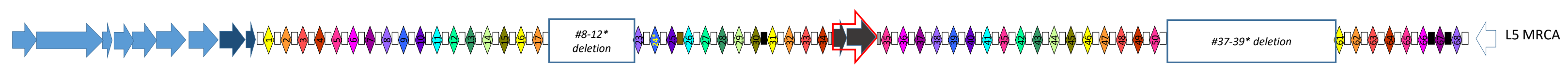

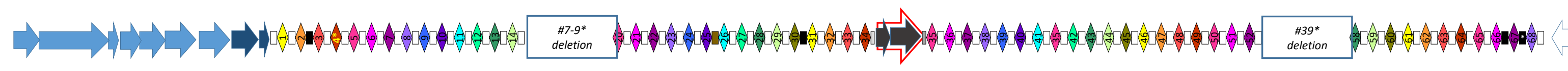

